\title{
Mit welchem Auflösungsgrad können Kompetenzen modelliert werden? In welcher Beziehung stehen Modelle zueinander, die Kompetenz in einer Domäne mit unterschiedlichem Auflösungsgrad beschreiben?
}

\author{
Knut Neumann
}

Zusammenfassung: Kompetenzmodelle sollen Kompetenzen in Teilkompetenzen gliedern und Ausprägungen, bezogen auf diese Teilkompetenzen, beschreiben. Für die Modellierung (und in der Folge die Messung) von Kompetenz ergibt sich damit die Frage, in wie viele Teilkompetenzen eine Kompetenz sinnvoll gegliedert werden kann und wie detailliert Ausprägungen gemessen werden sollen. Die Zahl der Teilkompetenzen (d.h. die Feingliedrigkeit) und die Detailliertheit der Ausprägungen (d.h. die Feinkörnigkeit) eines Kompetenzmodells definieren den Auflösungsgrad des Modells. Die bisherige Forschung im Bereich der Kompetenzmodellierung zeigt, dass für die Kompetenz in einer Domäne Modelle mit unterschiedlichem Auflösungsgrad zu finden sind. Hier stellt sich die Frage, in welcher Beziehung diese Kompetenzmodelle zueinander stehen. Der vorliegende Beitrag fasst die Ergebnisse der Diskussion innerhalb des Schwerpunktprogramms „Kompetenzmodelle zur Erfassung individueller Lernergebnisse und zur Bilanzierung von Bildungsprozessen“ bezüglich dieser Fragestellungen zusammen.

Schlüsselwörter: Kompetenzen · Kompetenzmodelle $\cdot$ Teilkompetenzen · Auflösungsgrad

\section{Up to which level of detail can a competence be described? Which relation do competence models describing a competence at different levels of detail have to each other?}

Abstract: Competence models are supposed to structure a competence into different sub-com-
petencies and to describe different levels of competence with respect to these sub-competencies.
In developing a competence model (and measuring the respective competence) the following
questions need to be answered: How many sub-competencies can be meaningfully differenti-
ated and at which grain size should different levels of competence be measured. The number
of sub-competencies and the grain size of the competence levels define the level of detail of a
competence model. Previous research in the field of competence modeling suggests that the same

(C) Springer Fachmedien Wiesbaden 2013

Prof. Dr. K. Neumann $(\bowtie)$

IPN - Leibniz-Institut für die Pädagogik der Naturwissenschaften und Mathematik, Universität Kiel, Olshausenstr. 62, 24118 Kiel, Deutschland

E-Mail: neumann@ipn.uni-kiel.de 
competence can be modeled at different levels of detail. This raises the question which relation such competence models have to each other. The following article presents the results of a discussion of these questions in scope of the priority program "Competence Models for Assessing Individual Learning Outcomes and Evaluating Educational Processes".

Keywords: Competence $\cdot$ Competence models $\cdot$ Grain size $\cdot$ Sub-competencies

\section{Mit welcher Feingliedrigkeit lassen sich Kompetenzen modellieren?}

Die Feingliedrigkeit eines Kompetenzmodells bezieht sich auf die Zahl der Teilkompetenzen, die im Modell unterschieden werden. Je höher die Zahl der Teilkompetenzen, desto feingliedriger das Kompetenzmodell. Eine theoretische Begrenzung der Feingliedrigkleit ergibt sich aus der zugrunde liegenden Definition von Kompetenz. So lässt sich nach Weinert (2001) Kompetenz als die Summe der Fähigkeiten und Fertigkeiten sowie der motivationalen, volitionalen und sozialen Bereitschaften eines Individuums verstehen, wie sie zur Lösung von Problemen in variablen Situationen benötigt werden. In diesem Sinne kann eine Kompetenz in Teilkompetenzen im Sinne derjenigen Fähigkeiten und Fertigkeiten sowie Bereitschaften gegliedert werden, wie sie zur Lösung von Problemen in bestimmten Teilbereichen einer Domäne benötigt werden. Die theoretische Grenze der Feingliedrigkeit ist dann erreicht, wenn zur Lösung eines Problems nur noch einzelne Fähigkeiten oder Fertigkeiten oder Bereitschaften benötigt werden. Eine einzelne Fähigkeit oder Fertigkeit stellt im Sinne der Definition von Kompetenz nach Weinert (2001) also keine Teilkompetenz mehr dar. Im Kontext der im Rahmen des DFG-Schwerpunktprogramms (SPP) „Kompetenzmodelle zur Erfassung individueller Lernergebnisse und zur Bilanzierung von Bildungsprozessen“ verwendeten Definition von Kompetenz (vgl. Klieme und Leutner 2006) ist die Begrenzung durch die Forderung nach Erlernbarkeit gegeben. Die Gliederung einer Kompetenz in Konstrukte, die nicht mehr durch gezielte Instruktion zu lernen sind, ${ }^{1}$ ist im Kontext dieses Kompetenzbegriffs ausgeschlossen. Praktisch wird die Gliederung von Kompetenzen in Teilkompetenzen durch die Messbarkeit begrenzt. Die Gliederung einer Kompetenz in Teilkompetenzen, die sich empirisch nicht eindeutig unterscheiden lassen, ist nur bedingt sinnvoll, zum Beispiel wenn die theoretisch begründete Annahme besteht, dass sich die jeweiligen Teilkompetenzen parallel entwickeln. Inwieweit sich Kompetenzen in Teilkompetenzen gliedern lassen, hängt dabei möglicherweise auch vom Stand der Kompetenzentwicklung in der untersuchten Stichprobe ab. So kann sich Kompetenzentwicklung sowohl als Veränderung der Kompetenzstruktur im Sinne einer Differenzierung in Teilkompetenzen als auch im Sinne einer Integration verschiedener Teilkompetenzen manifestieren (vgl. z. B. Gschwendtner 2011; Nickolaus et al. 2011).

\section{Mit welcher Feinkörnigkeit lassen sich Kompetenzen messen?}

Eine Kompetenz messen heißt zu erfassen, in welcher Ausprägung eine Kompetenz bei einem Individuum vorliegt. Die Messung einer Kompetenz setzt eine Skala voraus. Mithilfe dieser Skala lassen sich unterschiedliche Ausprägungen der Kompetenz erfassen. Die Feinkörnigkeit (grain size) einer Skala gibt dabei an, wie fein diese differenziert; je 
niedriger die Feinkörnigkeit, desto gröber die Skala. Umgekehrt gilt: Je höher die Feinkörnigkeit, desto feiner die Skala. Dabei beeinflusst die Feinkörnigkeit die Messmethodik und umgekehrt: Eine hohe Feinkörnigkeit erfordert Messinstrumente, die tatsächlich in der Lage sind, auf der Ebene dieser Feinkörnigkeit noch unterschiedliche Ausprägungen zu unterscheiden. Umgekehrt wird die Feinkörnigkeit dadurch begrenzt, inwieweit mit einem gegebenen Messinstrument noch unterschiedliche Ausprägungen messbar beziehungsweise empirisch trennbar sind. Grundsätzlich ist die Feinkörnigkeit mit der sich eine Kompetenz noch messen lässt nach der im SPP gewählten Definition von Kompetenz durch die Lernbarkeit definiert: Eine Erhöhung der Feinkörnigkeit einer Kompetenzmessung ist nur sinnvoll, wenn bei dieser Feinkörnigkeit auch eine Veränderung in der Ausprägung der Kompetenz als Folge von Instruktion erwartet werden kann.

\section{In welcher Beziehung stehen Modelle zueinander, die eine Kompetenzdomäne mit unterschiedlichem Auflösungsgrad erfassen?}

Im Auflösungsgrad eines Kompetenzmodells sind die Aspekte der Feingliedrigkeit und der Feinkörnigkeit der jeweiligen Kompetenz zusammengeführt. Der Auflösungsgrad eines Kompetenzmodells steigt einerseits mit der Feingliedrigkeit und andererseits mit der Feinkörnigkeit. Das heißt je mehr Teilkompetenzen ein Kompetenzmodell unterscheidet bzw. je mehr Ausprägungen unterschieden werden, desto höher ist der Auflösungsgrad des Kompetenzmodells. Entsprechend kann der Auflösungsgrad eines Kompetenzmodells durch die Ausdifferenzierung in mehr Teilkompetenzen und/oder die Ausdifferenzierung weiterer Ausprägungen erhöht werden.

Die Angemessenheit des Auflösungsgrades hängt dabei vom jeweiligen Anwendungskontext ab (vgl. Kauertz et al. 2008). Im Kontext von Large-Scale-Assessments, in denen die Leistungen von Proband(inn)en hinsichtlich allgemeiner Bildungsziele eingeschätzt werden sollen, können wenige, breit gefasste Teilkompetenzen und Ausprägungen ausreichend sein. Auf der Unterrichtsebene werden differenziertere Unterscheidungen benötigt. So lassen sich aus Kompetenzmodellen mit niedrigem Auflösungsgrad nur bedingt Informationen für eine systematische und gezielte Entwicklung von Kompetenzen ableiten. Für eine Unterscheidung von Proband(inn)en(gruppen) in experimentellen Studien sind hoch auflösende Kompetenzmodelle sogar eine wichtige Voraussetzung. Denn auf kurzen Zeitskalen, wie sie in experimentellen Interventionen häufig zu finden sind, können weder Zuwächse im Bereich globalerer Kompetenzen wie zum Beispiel naturwissenschaftlicher Kompetenz noch bezüglich globalerer Ausprägungen wie zum Beispiel konzeptuellen Verständnisses im Gegensatz zu Faktenwissen erwartet werden (vgl. Viering et al. 2010).

Kompetenzmodelle mit unterschiedlicher Auflösung stehen idealerweise in einer hierarchischen Beziehung zueinander. Das heißt: In einem untergeordneten Modell wird eine Kompetenz oder Teilkompetenz in weitere Teilkompetenzen ausdifferenziert oder Ausprägungen einer Kompetenz oder Teilkompetenz in weitere Ausprägungen. Umgekehrt fasst ein übergeordnetes Modell Teilkompetenzen und/oder Ausprägungen zusammen. Dabei gilt: Kompetenzmodelle unterschiedlicher Auflösung, die zueinander in Beziehung stehen, lassen sich einfacher aufeinander beziehen, wenn sie systematisch aufeinander bezogen entwickelt wurden. Das heißt: Differenzierte Modelle mit höherem Auflö- 
sungsgrad sollten aus allgemeineren Modellen mit niedrigerem Auflösungsgrad durch Unterscheidung von Teilkompetenzen oder Differenzierung von Ausprägungen in feiner abgestufte Ausprägungen gewonnen werden.

Grundsätzlich lassen sich auch Kompetenzmodelle denken, die dieselbe Kompetenz mit unterschiedlicher Auflösung beschreiben, jedoch nicht in einer hierarchischen Beziehung zueinander stehen. Das hängt vom zugrunde liegenden Kompetenzbegriff und von der jeweiligen Domäne (und den fachlichen Theorien dieser Domäne) ab. So ist bei der Modellierung von Kompetenzen auch zu berücksichtigen, dass Kompetenzen einer Domäne (z. B. Naturwissenschaft) mit Kompetenzen anderer Domänen (z. B. Lesen) interagieren.

Die Modellierung von Kompetenzen sollte (stärker als bisher) das nomologische Netzwerk, in dem sich die betreffende Kompetenz befindet, berücksichtigen. Das heißt: Kompetenzmodelle mit hoher Auflösung sollten explizit in übergeordnete Kompetenzmodelle eingeordnet werden; umgekehrt sollten Kompetenzmodelle mit einem sehr breiten Fokus hinsichtlich ihrer Binnenstruktur beschrieben werden. In beiden Fällen sollten die Beziehungen der jeweiligen Kompetenzmodelle zueinander, das heißt die nomologische Struktur der Kompetenz, empirisch geprüft werden. Entsprechende Untersuchungen stellen bisher die Minderheit der Untersuchungen im Bereich der Kompetenzmodellierung dar.

Danksagung: Diese Veröffentlichung wurde ermöglicht durch Sachbeihilfen der Deutschen Forschungsgemeinschaft (Kennz.: NE 1368/2-1, 2-2 und 2-3) im Schwerpunktprogramm „Kompetenzmodelle zur Erfassung individueller Lernergebnisse und zur Bilanzierung von Bildungsprozessen“" (SPP 1293).

\section{Anmerkung}

1 Prinzipiell können elementare Hirnfunktionen auch als erlernbar aufgefasst werden, möglicherweise können sie auch durch Instruktion verändert werden - nicht jedoch gezielt.

\section{Literatur}

Gschwendtner, T. (2011). Die Ausbildung zum Kraftfahrzeugmechatroniker im Längsschnitt. Analysen zur Struktur von Fachkompetenz am Ende der Ausbildung und Erklärung von Fachkompetenzentwicklungen über die Ausbildungszeit. In R. Nickolaus \& G. Pätzold (Hrsg.), Lehr-Lernforschung in der gewerblich-technischen Berufsbildung (25. Beiheft der Zeitschrift für Berufs- und Wirtschaftspädagogik, S. 55-76). Wiesbaden Franz Steiner Verlag.

Kauertz, A., Fischer, H. E., Lau, A., \& Neumann, K. (2008). Kompetenzmessung durch Leistungstests. Der mathematische und naturwissenschaftliche Unterricht, 61, 75-79.

Klieme, E., \& Leutner, D. (2006). Kompetenzmodelle zur Erfassung individueller Lernergebnisse und zur Bilanzierung von Bildungsprozessen. Beschreibung eines neu eingerichteten Schwerpunktprogramms der DFG. Zeitschrift für Pädagogik, 52, 876-903.

Nickolaus, R., Geißel, B., Abele, S., \& Nitzschke, A. (2011). Fachkompetenzmodellierung und Fachkompetenzentwicklung bei Elektronikern für Energie- und Gebäudetechnik im Verlauf der Ausbildung - Ausgewählte Ergebnisse einer Längsschnittstudie. In R. Nickolaus \& G. Pätzold (Hrsg.), Lehr-Lernforschung in der gewerblich-technischen Berufsbildung (25. Beiheft der Zeitschrift für Berufs- und Wirtschaftspädagogik, S. 77-94). Wiesbaden Franz Steiner Verlag. 
Viering, T., Fischer, H. E., \& Neumann, K. (2010). Die Entwicklung physikalischer Kompetenz in der Sekundarstufe. In E. Klieme, D. Lautner \& M. Kenk (Hrsg.), Kompetenzmodellierung Eine aktuelle Zwischenbilanz des DFG-Schwerpunktprogramms (56. Beiheft der Zeitschrift für Pädagogik, S. 92-103). Weinheim: Beltz.

Weinert, F. E. (2001). Concept of competence: A conceptual clarification. In D. S. Rychen \& L. H. Salganik (Hrsg.), Defining and selecting key competencies (S. 45-65). Seattle: Hogrefe. 